Journal of Engineering and Applied Sciences 14 (Special Issue 1): 3891-3897, 2019

ISSN: 1816-949X

(C) Medwell Journals, 2019

\title{
Effiecient Versatile e-Learning Framework Based on Personality Traits and Kolb's Learning Style
}

\author{
${ }^{1}$ Upadhyay Anand Trilokinath and ${ }^{2}$ Santhosh Kumar Singh \\ ${ }^{1}$ Department of Information Technology, AMET University, Chennai, India \\ ${ }^{2}$ Department of Information Technology, Tagore College of Science and Commerce, Mumbai, India
}

\begin{abstract}
In the current world of technology, there exists enormous amount of data emerging every day, thus, the data needed to be utilized for a better purpose in business. The businesses like e-Commerce, banking, social media, e-Learning, etc., found useful with the mining techniques to mine the data to gather useful information from it. In the peak of e-Learning transmission in the instructive information mining field, there contains the effective increase of versatile and smart electronic mentors, instructive applications and tools and based on the tools and with applications mining frameworks in instructive information. The study proposes efficient web based versatile e-Learning framework. The study focuses on understanding the relationship between learner's learning style and their personality. The research utilized information mining procedures added with learning styles, i.e., Kolb's experiential learning styles. The main objective of the research included in deciding the best showing design for every learning participant based on their personality traits. The framework ensures the system is accessible everywhere and at anytime in web. It additionally includes the interesting features like learning recordings, versatile introductions and several tests for understudies. The research is produced for both educators and understudies for choosing the best learning process and accomplishes scholastic rates.
\end{abstract}

Key words: e-Learning, smart electronic mentor, learning style, Kolb's experiential learning styles, web, understudies

\section{INTRODUCTION}

The learning industry obtained a new revolution with the help of Internet called e-Learning that changed the training and education industry into a new trend in learning because of its available features, uninterrupted online service with any time any where accessibility. The concept was introduced by the American Society for Training and Development in 1996 at the Internet Based Training workshop. Later the e-Learning had gone into a boom and bust cycle of development. The adaptive e-Learning follows the principle that every student is unique in sense like background, educational needs, different in interests, capacity in learning, etc. The aim of adaptive e-Learning is with the support of Kolb's experiential model to find out individual learner's preferred learning style based on the big five personality traits. Finding out the useful information like uniqueness and transforming that into useful content and appropriate training processes that are relevant to every individual student.

Information mining is a part of e-Learning frameworks that finds concealed and useful information, guidelines and analysis through the way towards by breaking down the useful data that affects largely in the research field. There are lots of research is undergoing in the field of information mining. The information mining strategies are identified and utilized making the learning process enhanced and additionally use the features provided in the e-Learning frameworks. These things extract the poor scholastic understudies related with the end goal for obtaining the better execution. From the emergence of internet from late 1980's the e-Learning also started to get boom and topped the research trending topics and numerous researchers built many methodologies. Information mining field in e-Learning has enormous techniques in it that extracts the useful information from the enormous data.

The e-Learning framework works as dynamic learning environment which is made possible with the help of internet that makes the learning procedure more effective by establishing learners to make it available with the accessible assets and several administrations. Currently, Insightful learning frameworks consider challenging amongst the field of training. In e-Learning there exists a supporting subject called Instructive information mining which viewed as a persuading subject that creates the methods and systems in perceiving the effective

Corresponding Author: Upadhyay Anand Trilokinath, Department of Information Technology, AMET University, Chennai, India 


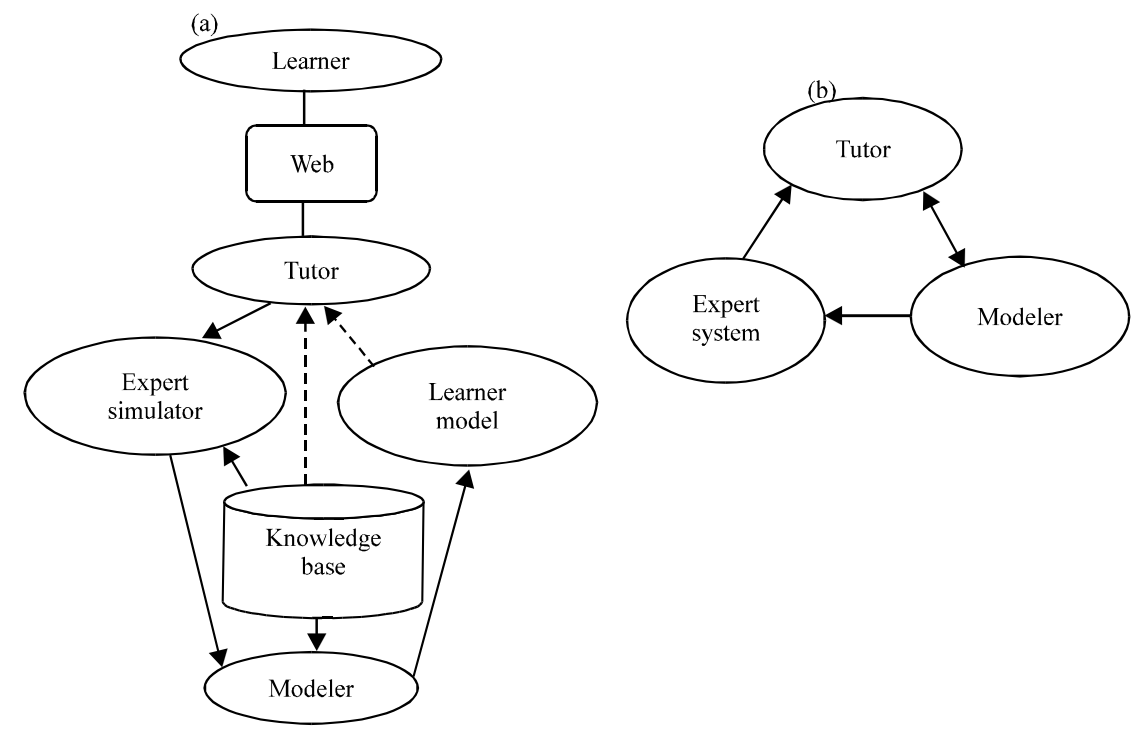

Fig 1: a) Intelligent e-Learning system and b) Intelligent tutor systems

information which will upgrade the instructive procedure in the learning process. In the utilization of those methods and techniques, a well established framework is created. Along with it any changes in the instructive execution also been accomplished that evaluates the understudies learning procedure effectively.

Intelligent e-Learning system: The study utilizes intelligent e-Learning system that generates the possible solution for the problem. Intelligent e-Learning systems are considered as a new framework inside the e-Learning system with the basic principles identical between it. But it eliminates the learner generalization with the individual access towards the learner's study which is so-called as intelligent principles. This makes the learner's study more effective. There exits many methods in implementing the intelligent principles, selecting the appropriate solution from a set of intelligent principles based on the studying material form of study course etc.

The research discuss mainly used principle called adaptability principle where it explains the learner's study progress and make appropriate actions. The intelligent learning system flows in the process of learner approaching the suitable model. The learner first starts search for the effective e-Learning materials in the web. The e-Learning material is spotted effective by various facts like coaching skills, understandability, content coverage, material quality, other than this the quality is identified by other various measures like comments, user ratings, likes and dislikes etc. The learner chooses the appropriate tutor once the learner found satisfied which was clearly defined in Fig. 1a. The tutor uses expert simulator that connects with the modeler and knowledge base. From the model the learner model predicts the best effective leaner model to the tutor. That predicts the system appropriate solution for it. The intelligent tutor system predicts the appropriate modeler for the expert system which shown in Fig. 1b.

Kolb learning style: From the knowledge of Experiential Learning Theory (ELT) Model, Kolb developed its own Learning Style Inventory (LSI) which is a self-assessment tool that determines an individual learner's preferred learning style. The learning style tool was developed to address the issues like increasing the individual learner understands their preferred learning style and validating the ELT and investigating the characteristic features of individual's learning styles. The understanding of student's learning styles benefits both learners and educators. For learners it would help in understanding the strengths and weaknesses apart from their preferred learning style that aims in maximizing their learning potential with obtaining the most out of their experiences. For educators it helps in evaluating their teaching methodologies and selection of learning opportunities that match their students (e.g., indulging variety of teaching strategies, methodologies, to match their student's preferences and styles). The research suggests that providing a match between teaching and learning styles will benefit student's learning process in higher. Several meta-analyses have found that customized training provided to student's learning styles have improved students in academia and improved the attitude towards learning. Even though students can now master 


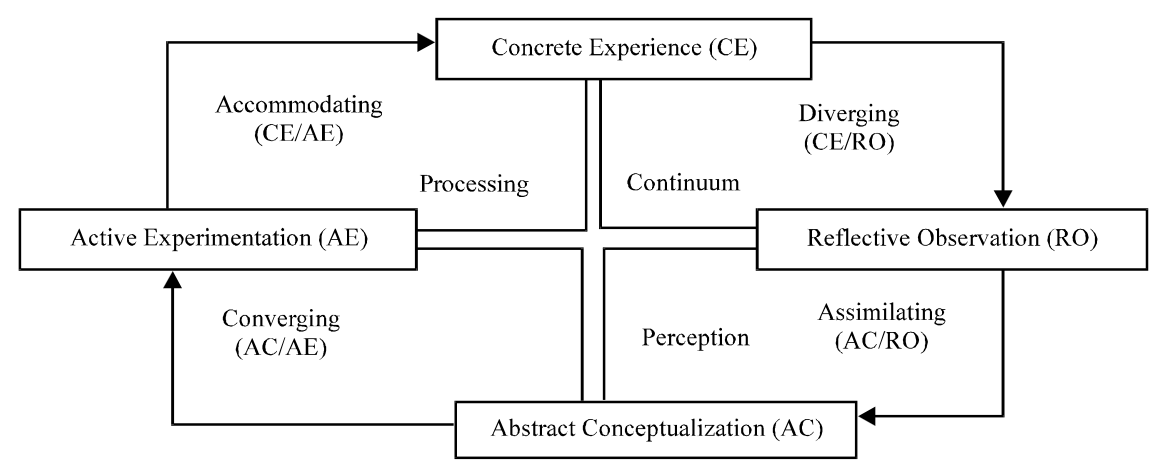

Fig. 2: Kolb's experiential learning model

easy information despite of their learning style which in turn they learn effectively and in rapidly when using their preferred learning style.

In several existing systems identified the preferred learning and teaching styles of students using the Myers-Briggs Type Indicator (MBTI) and the benefits accommodated with matching those styles. The research's literature review found several facts that very few studies have been researched on the learning styles over any specific study based. Therefore, the aim of the research is to identify the foremost learning style among online based interested students using Kolb's LSI and to examine the association with gender, academic achievement, clinical experience level and specialty interest.

Willcoxson and Prosser stated that the Kolb's experiential learning which was built with the four learning modes with two bipolar learning dimensions which is concrete or in abstract based (the perceiving axis as shown in Fig. 2 and active or reflective (the processing axis as shown in Fig. 2. From a view of hypothetical point of view, it is considered that any learner will tend to consciously move through every mode in the learning cycle. Nonetheless, many researches on the subject have depicted that it's not assured that every learners equally experience same like other learners as well as each stage of this cycle. The learner's preferences that rise during the stages of the cycle do not affect them as better or worse learners. In general each individual preferences differs in learning style that resulting from capability of learn through experiencing which can be framed as Concrete Experience (CE) or the construction of appropriate theoretical frameworks which can be said as Abstract Conceptualization (AC) that combined with the ability to either learn by doing with Active Experimentation or implementation $(\mathrm{AE})$ or through the concept of reflection by Reflective Kbservation (RO).

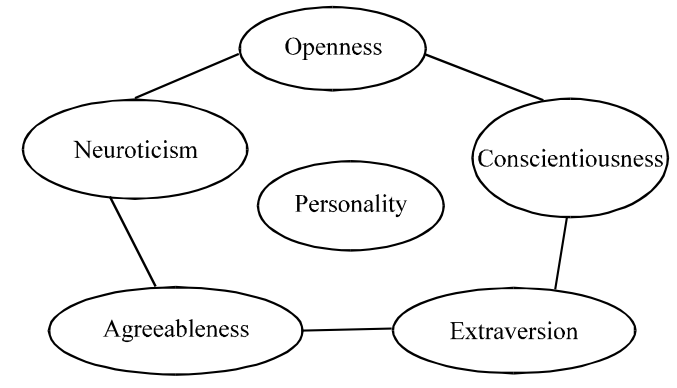

Fig. 3: The big five personality traits

The big five personality factors: The personality can be defined as individual's though pattern, emotion and behavior put together with psychological mechanisms that identify the characteristic of a person. The most popular way of representing any personality is by big five personality factors. The big five personality traits are neuroticism (worried, anxious, emotionally unstable and insecure), extraversion (sociable, talkative, active and cheerful), openness (imaginative, curious, insightful, broad-minded and original), agreeableness (caring, altruistic, kind, sympathetic and supportive) and conscientiousness (thorough, responsible, careful, self-disciplined and organized) which is as shown in the Fig. 3. The theory helps in many places in current world for instance selecting the employee during interview process, performance analysis, etc.

Other traits: The personality of the learners other than the big five traits it also included the characteristics of the learners by considering gender, country born, age, education, job function, job level. This derives the results through the facts in association with the Kolb's experiential styles.

Literature review: Onah and Sinclair (2015) introduced a learning structure an adaptive learning framework that utilized in investigation of an popular adaptive Massive 
Open Online Courses (MOOC) system from personalized learning approach. It addressed the prevalent issues like high dropout rates under the online learning system that occurred in MOOC. Zaiane (2002) presented recommendation agent called e-Learning task recommender that recommended the learning task for a learner based on the learner tasks and their successes also with the tasks based on the other similar learners. Monahan et al. (2008) presented a CLEV-R Model, a Collaborative Learning Environment with Virtual Reality. The presented model considered web-based system that utilized Virtual Reality (VR), multimedia and provided several communication tools that made collaboration among students.

Kimmons and Veletsianos (2018) investigated public Internet Data Mining (IDT) methods that explained the individuals to engage in a broader selection methods. The IDT generally involves multidisciplinary collaboration among subject matter experts, instructional designers, assessment experts and several subject faculties that created a educational intervention. Nebel et al. (2016) involved four data mining approaches namely $\mathrm{k}$-Nearest Neighbour (k-NN), Decision Tree (DT), Naive Bayes (NB) and Neural Network (NN) that analyzed the issue of dropout students. Those four techniques trained and tested with 10 -fold cross validation. With the support of Genetic Algorithm (GA) related with feature selection method, technologies like online self-efficacy, online learning readiness and previous online experience were found as the most important factors in predicting the dropouts. Zeiler (2012) presented per-dimension based learning rate method to the gradient descent called ADADELTA. The presented method dynamically adapted the time with only first order information and provided the minimal computational overheads in beyond to the vanilla stochastic gradient descent method. Janet (2018) presented a versatile e-Learning framework that incorporated an outstanding canny electronics English e-Learning mentorship with the information mining procedures. The framework decided the appropriate showing pattern for every learner. Ruder (2016) provided a reader with intuitions by studying the behavior of different algorithms like gradient descent that performs optimization problem and also utilized for black-box optimizers.

Khan and Sahai (2012) selected two different gradient descent algorithms called back propogation and Levenberg-Marguardt and three population based heuristic algorithm called Bat algorithm, particle swarm optimization and Genetic algorithm that established the advantages of new meta-heuristic bat algorithm as better compared to other algorithms in context to the e-Learning.
Riedmiller (1994) presented a improvement model back propagation for training of weights in feed-forward neural network. It introduced the concept of supervise learning in the multi-layer preceptor based on the popular technique called gradient descent. The research addressed the drawbacks in the original back propagation learning procedure. Villaverde et al. (2006) presented an approach that recognizes automatically the learning styles of individual students according to the actions that he or she has performed in an e-Learning environment. This recognition technique is based upon feed-forward neural networks. Mangina and Kilbride (2008) examined the applicability of the IUI techniques on the online eLearning environment. In addition the research also made use of the user modeling techniques, extraction techniques, information mining and retrieval techniques and few collaboration filtering methods. The research recommended the documents that tend to encourage the users in widening the knowledge on a given topic or reinforce the existing knowledge. De-Marcos et al. (2017) presented an approach on social gamification and several set of tools designed in addressing the situated motivations in affordances of students (relatedness, autonomy and competedness) in gamification. Gamification is generally a game mechanics and game design in a non-game contexts that engaged users towards a motivate action. Albatayneh et al. (2018) introduced a recommendation system that recommended interesting post messages on learners in an e-Learning online discussion forum related to the semantic content-based filtering and learner's negative ratings. The research evaluation was made on the e-Learning recommender system towards the existing recommender system on e-Learning that utilized similar filtering techniques addressed with recommendation accuracy and learner's performance. Albatayneh et al. (2014) enhanced the recommendation system to the relevant of post messages to users based on to the content-based filtering and several latent semantic analysis that increased the dynamic nature in online forums that helped users in discovering the relevant post messages and it prevented from redundant post messages and specific bad content post messages.

Traditional learning methods and web based learning: In 2001, Weber and Brusilovsky proposed ELM-ART. ELM-ART is an astute and intelligent instructive framework that plans to bolster figuring out how to program in LISP. It helped instructors and executives to have a superior comprehension of the system of instructive information mining and how information mining procedures function to bolster the decision making 
procedure. A versatile e-Learning framework was taken after where each time an understudy enters the application another arrangement of issues will show up. What's more, instructors help understudies as indicated by their advance through modified assignments in view of their experience, for instance, a correlation between conventional techniques is showing and e-Learning based strategies. An e-Learning framework was executed on undergrad young lady understudies and found that utilizing e-Assets make understudies more appealing and give them a superior seeing, particularly on account of concentrate electronic gadgets and segments and in addition doing research center examinations. Scientists utilized the electronic frameworks not exclusively to streamline the instructing and the learning procedure, additionally to enhance it by utilizing information mining strategies and apparatuses.

\section{MATERIALS AND METHODS}

The versatile English e-Learning electronic mentor is a versatile online framework targets second rate class understudies. It can characterize the learner style, the reasonable substance for learning and a versatile introduction method.

The framework refreshed consequently as per the learner trademark and conduct. Versatile e-Learning electronic mentor engineering comprises of 3 principle layers as appeared in Fig. 4. In which the primary layer is the database layer in which every one of the practices of the understudy were spared. The second layer is the application layer contains the learning recordings, versatile introductions and also tests. The last layer is the customer layer that incorporates the understudies and the framework executive. In the customer layer, customers can open the adaptive English e-Learning electronic guide Every where and in addition Every Time (EWET). The proposed adaptive e-Learning electronic coach segments are portrayed in the following subsections. Moreover, the flowchart of proposed framework in which the understudies at first take the pre-survey (Kolb's experiential learning style) then the understudies will be grouped into five fundamental bunches as per their outcomes in the pre-questionnaire.

Kolb's Model: The Kolb's Model merges two bi-polar features called abstract and concrete feature ranges on dealing towards the theoretical concepts tend to dealing over tangible objects while grasping new experiences. The other active and reflective combined features range from direct participation that tends to detached observation while transforming experiences. The 4-featured cycle of

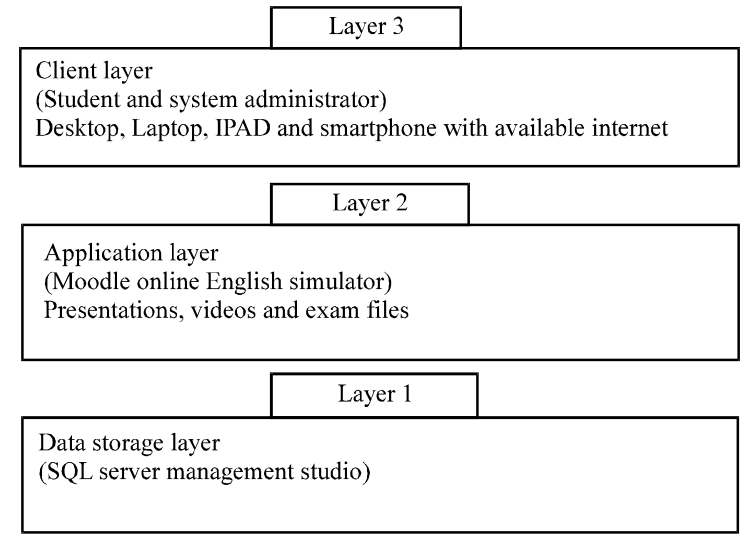

Fig. 4: The proposed adaptive e-Learning web-based tutor architecture

Kolb's learning style is presented in Fig. 2 where instant Concrete Experience (CE) is responsible for Observation and Reflection (RO) where the experience is further assimilated into Abstract Conceptualization (AC) and that formed into Active Experimentation (AE). Active Experimentation $(\mathrm{AE})$ whole some the cycle of learning and assures that it starts anew from assistance in the creation of new experiences.

Learning style: Learning style was measured with the support of the latest version of LSI known to be (KLSI 3.1). The KLSI 3.1 Model version is a forced-choice where it took data from Kolb and Kolb (2005) 12-item inventory ranked based on the individual's relative choice preferences in the 4 learning modes-Abstract Conceptualization (AC), Concrete Experience (CE), Reflective Observation (RO) and Active Experimentation (AE).

Personality: The NEO-FFI association published by psychological assessment resources, incorporation employed to assess these big five factors of personality. It data taken is rated on a 5-point scale. In the study, the reliability estimates were obtained $0.81,0.74$, $0.62,0.69,0.81$ for neuroticism, extraversion, openness, agreeableness and conscientiousness respectively.

Principle of adaptability: The principle of adaptability brings the support for individual access in learners study. The principle considered information about learner study progress to store in developed model and the information tells system rules about the manageability of teaching materials from data repository and then these materials served to specific learner. Once the materials are been invoked a change in learner study progress, then the information is again tended to store in the student model. 


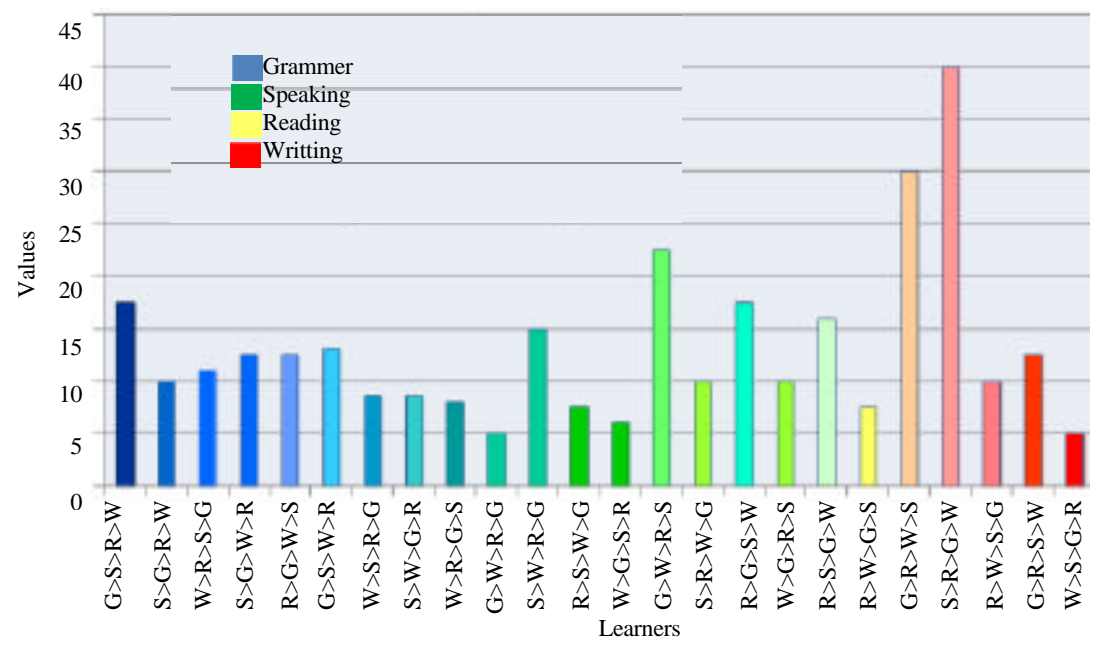

Fig. 5: Patterns analysis vs. number of students

Table 1: Analy sis of the Kolb's Model with big five personality traits

\begin{tabular}{|c|c|c|c|c|}
\hline Variables & Concrete Experience (CE) & Reflective Observation (RO) & Abstract Conceptualization (AC) & Active Experiment (AE) \\
\hline Gender & 0.15 & 0.03 & -0.30 & 0.13 \\
\hline Country of birth & -0.04 & -0.10 & 0.01 & 0.13 \\
\hline Age & 0.09 & 0.05 & 0.02 & -0.15 \\
\hline Education & -0.05 & 0.00 & 0.03 & 0.03 \\
\hline Job function & -0.02 & 0.11 & -0.04 & -0.06 \\
\hline Job level & 0.06 & -0.20 & 0.10 & 0.03 \\
\hline Neuroticism & -0.01 & -0.02 & -0.02 & 0.05 \\
\hline Extraversion & 0.28 & 0.31 & -0.21 & 0.27 \\
\hline Openness & 0.03 & 0.01 & 0.07 & -0.11 \\
\hline Agreeableness & -0.15 & 0.05 & 0.07 & 0.01 \\
\hline Conscientiousness & -0.29 & 0.03 & 0.13 & 0.12 \\
\hline $\mathrm{R}^{2}$ & 0.16 & 0.14 & 0.17 & 0.15 \\
\hline $\mathrm{F}$ & 4.42 & 3.69 & 4.79 & 4.04 \\
\hline
\end{tabular}

\section{RESULTS AND DISCUSSION}

In the research, five Jordanian's schools were visited by the research team in order to implement the developed approach and collecting the data from students. The total number of participant's students is 314 students with both gender all of them with the same age. The data is trained into the rapid minor as training set and then divided using cross-validation into training and testing set. A RapidMiner is a kind of powerful data mining tool that supports in rapidly building predictive analytic workflows. It provides an integrated environment for data preparation, machine learning, text mining, deep learning and predictive analytics. It conducted two experiments as follows. The experiment denoted is students Behaviors patterns analysis that aims to check which pattern was the most chosen pattern by the students. The RapidMinor results for analyzing student's behavioral patterns (Fig. 5). The experimental results shows the pattern (Speaking-reading-grammar-writing) is the most chosen pattern by the students.

The results of the study contribute in the understanding of individual differences with reference to the personality of the learners and Kolb's experiential learning style that is been investigated the data. From the analysis contain a results of the multiple regression analyses of the relationship between 5 factor personality traits and four experiential learning modes. As shown in Table 1 extraversion was positively related to concrete experience and negatively related to abstract conceptualization. Extraversion was also positively related to active experiment and negatively related to reflective observation. Results have indicated that the best personality trait that appropriate to the Kolb's experiential learning cycle is considered to be extraversion. Extraverted individual's dominant in the learning styles were said to be accommodator rather than diverger, converger or assimilator.

\section{CONCLUSION}

Numerous mining systems can be utilized to upgrade the learning and instructing forms. The study proposed an adaptive e-Learning English electronic mentor that offers numerous instructive offices, for example, recordings, introductions and tests to furnish the 
understudy with versatile and appealing learning conditions. The study examined the relationship between the five factor personality and Kolb's experiential learning style. The results have indicated that one among the 5 personality traits have been is been associated with Kolb's Model, i.e., extraversion. Overall the Kolb's Model constructed and argued towards the uniquely distinct apart from personality. The study examined the relationship between five factor personality and experiential learning style. Our results indicate only one of the five factor personality traits-extraversion is associated with experiential learning style. Overall, Kolb's experiential learning style construct is argued to be uniquely distinct from personality.

The second exploration means to discover the best understudy accomplishment design and the outcomes demonstrates that it is the example (talking-perusingsyntax-composing) which its scores are $87.4 \%$ and higher. To finish up, the proposed adaptive e-Learning English online mentor can help both instructors and understudies to take after the best learning process and accomplish the most astounding scholastic evaluations.

\section{RECOMMENDATIONS}

In future, it is a progressing research, the study want to extend the current research to test the impact of utilizing EDM procedures on understudies learning procedure and a few components will be added to make the coach shrewd.

\section{REFERENCES}

Albatayneh, N.A., K.I. Ghauth and F.F. Chua, 2014. A Semantic Content-Based Forum Recommender System Architecture Based on Content-Based Filtering and Latent Semantic Analysis. In: Recent Advances on Soft Computing and Data Mining, Herawan, T., R. Ghazali and M.M. Deris (Eds.). Springer, Cham, Switzerland, ISBN:978-3-319-07691-1, pp: 369-378.

Albatayneh, N.A., K.I. Ghauth and F.F. Chua, 2018. Utilizing learners' negative ratings in semantic content-based recommender system for e-Learning forum. J. Educ. Technol. Soc., 21: 112-125.

De-Marcos, L., A. Garcia-Cabot and E. Garcia-Lopez, 2017. Towards the social gamification of e-Learning: A practical experiment. Intl. J. Eng. Educ., 33: 1-14.

Janet, J.C., 2018. Intelligent web-based English E-learning tutor with data mining technique. Indian J. Public Health Res. Dev., 9: 436-439.
Khan, K. and A. Sahai, 2012. A comparison of BA, GA, $\mathrm{PSO}, \mathrm{BP}$ and LM for training feed forward neural networks in e-Learning context. Intl. J. Intell. Syst. Appl., 4: 23-29.

Kimmons, R. and G. Veletsianos, 2018. Public internet data mining methods in instructional design, educational technology and online learning research. TechTrends, 62: 492-500.

Kolb, A.Y. and D.A. Kolb, 2005. The Kolb learning style inventory-Version 3.12005 technical specifications. Hay Resource Direct, Massachusetts. http://www.whitewater-rescue.com/support/ pagepics/sitechmanual.pdf.

Mangina, E. and J. Kilbride, 2008. Evaluation of keyphrase extraction algorithm and tiling process for a document/resource recommender within e-Learning environments. Comput. Educ., 50: 807-820.

Monahan, T., G. McArdle and M. Bertolotto, 2008. Virtual reality for collaborative e-learning. Comput. Edu., 50: 1339-1353.

Nebel, S., S. Schneider and G.D. Rey, 2016. Mining learning and crafting scientific experiments: A literature review on the use of minecraft in education and research. J. Educ. Technol. Soc., 19: 355-366.

Onah, D.F. and J.E. Sinclair, 2015. Massive open online courses: An adaptive learning framework. Proceedings of the 9th International Conference on Technology, Education and Development (INTED2015), March 2-4, 2015, University of Warwick, Madrid, Spain, ISBN:9788460657637, pp: 1258-1266.

Riedmiller, M., 1994. Advanced supervised learning in multi-layer perceptrons-from backpropagation to adaptive learning algorithms. Comput. Stand. Interfaces, 16: 265-278.

Ruder, S., 2016. An overview of gradient descent optimization algorithms. Mach. Learn., 1: 1-14.

Villaverde, J.E., D. Godoy and A. Amandi, 2006. Learning styles recognition in e-Learning environments with feed-forward neural networks. J. Comput. Assisted Learn., 22: 197-206.

Zaiane, O.R., 2002. Building a recommender agent for e-Learning systems. Proceedings of the International Conference on Computers in Education, December 3-6, 2002, IEEE, Auckland, New Zealand, ISBN: 0-7695-1509-6, pp: 55-59.

Zeiler, M.D., 2012. ADADELTA: An adaptive learning rate method. Mach. Learn., 1: 1-6. 